\title{
New spindle morphogenesis model by Dynein, Nudel, and the spindle matrix
}

\author{
Wei-Lih Lee ${ }^{1}$, Patricia Wadsworth ${ }^{1}$ \\ 'Department of Biology, University of Massachusetts, Amherst, Massachusetts 01003, USA \\ Cell Research (2009) 19:529-531, doi: 10.1038/cr.2009.49; published online 4 May 2009
}

It is well established that the mitotic spindle, the organelle responsible for chromosome segregation during mitosis, is built from microtubules, motor proteins, and associated structural and regulatory molecules. More controversial is the existence and identity of non-microtubule spindle components, collectively referred to as the matrix. What might be the role of a nonmicrotubule matrix? A static matrix could bind motor proteins, and generate motion of microtubules. This could be important for spindle assembly and function, because unlike the situation in interphase cells, where microtubule motors move along relatively static microtubule tracks, in the spindle, microtubules themselves move poleward, and move relative to each other during spindle elongation. A matrix could also contribute to the organization of spindle poles and/or to the distribution of spindle assembly factors $[1,2]$.

The existence of a non-microtubule spindle matrix is supported by the observation that following microtubule disassembly a fusiform structure, the 'spindle remnant' or 'spindle matrix', can be identified. In particular, early work showed that the microtubule motor protein kinesin [3] remained bound

Correspondence: Patricia Wadsworth

E-mail: patw@bio.umass.edu to the non-microtubule 'remnant' following calcium-induced microtubule disassembly of sea urchin spindles, consistent with the view that the matrix binds motor proteins [3].

Additional support for the idea that non-microtubule components contribute to spindle assembly and function is provided by ultrastructural analysis of spindles from diverse cells, which clearly shows that membrane components, perhaps elements of the endoplasmic reticulum or nuclear envelope, surround and invade the spindle. Membranes, and proteins associated with membranes, could provide an anchoring system for molecular motors. In addition, membranes could regulate ion fluxes in the spindle, and the surrounding membranes may form a diffusive boundary between the spindle and the cytoplasm [4]. In Drosophila, a spindle-shaped structure associated with the nuclear envelope is observed prior to spindle formation. This structure, which is composed of the proteins Skeletor, Megator, and Chromator, apparently assembles in the absence of microtubules [2]. Furthermore, recent studies in live Drosophila S2 cells showed that this structure is highly dynamic, adopting a morphology coalescent with spindle microtubules throughout mitosis [5].

Despite these observations, the identity of the matrix, and its role(s) in mitosis, have remained controversial. Progress in defining the spindle matrix was reported in 2006 by the laboratory of Yixian Zheng, who showed that a component of the nuclear lamina, lamin $\mathrm{B}$, is associated with mitotic spindles in frog extracts and human cells and is required for spindle assembly and function [6]. Lamin B staining was retained following microtubule disassembly, and this retained structure, which they called the LB3 matrix, also contained spindle assembly factors, including XMAP215, Eg5 and NuMA, but not TPX2, and was disrupted by the addition of detergent. The fact that the lamin B matrix contained spindle assembly factors and was composed at least in part of membranes and proteins associated with the nuclear envelope was consistent with earlier work, and suggested that it was the long sought spindle matrix.

In a new paper published in Nature Cell Biology, Ma et al. extend these studies of the LB3 spindle matrix, and now report that isolated matrix contains cytoplasmic Dynein and Nudel [7]. Nudel, also called NudE-like, has emerged as a conserved key regulator of cytoplasmic Dynein function. Genetic interactions between Nudel and Dynein were discovered in studies of Nudel homologues in Aspergillus nidulans (NudE) and Saccharomyces cerevisiae (Nd11) [8, 9]. NUDE was 
first identified as a multi-copy suppressor of $n u d F$, a conditional mutation of LIS1, in A. nidulans [8]. Mammals have two NudE homologues, Nudel (described above) and NudE, both of which interact with LIS1 and Dynein to regulate a diverse array of Dyneindependent cellular processes $[10,11$, $12,13]$. The new article by Ma et al. showed that Nudel also interacts with Lamin B to facilitate the assembly of LB3 matrix in a Dynein-dependent manner.

To examine the contribution of Dynein and Nudel to the formation of the LB3 matrix, Ma et al. assayed spindle formation in Xenopus egg extracts using Aurora A coated beads to stimulate microtubule formation. In control extracts, the beads generate asters, with long microtubules; with time the asters are replaced by 'balls', with a high density of short microtubules near the bead, and eventually spindles, composed of two beads and associated microtubules [7].

Addition of an N-terminal Nudel construct, or depletion of Nudel from the extract, blocks the formation of balls and spindles, resulting in only aster formation. This phenotype can be rescued by adding back purified Nudel. Interestingly, inhibition or depletion of either Nudel or Dynein reduces the intensity of LB3 along astral microtubules and blocks the formation of balls and spindles, suggesting that Dynein and Nudel both contribute to enrichment of LB3 on the microtubules and the proper formation of spindle structures. Immunofluorescence shows that both Nudel and Dynein co-localize with the LB3. Similar results were obtained using sperm to drive spindle formation in the extracts.

Somewhat surprisingly, when LB3 was depleted from the extract, the Dynein/Nudel matrix was still observed, suggesting that in addition to contributing to the formation and existence of an LB3 matrix, Dynein and Nudel are also part of the matrix. However, depletion of LB3 did not prevent 'ball' and spindle formation, but resulted in spindles that were $\sim 2$-fold longer and 'balls' that were of greater diameter, than observed in controls. These results suggest that proper assembly of the matrix regulates the size and length of the microtubule structures that form. Although the mechanism by which LB3 regulates the size of microtubule structures is not known, it is consistent with previous work showing that inhibition of Dynein in Xenopus extracts results in a long spindle phenotype [14].

The studies by Ma et al. provided new insights into the assembly of lamin B matrix (Figure 1). They show that spindle assembly factors Dynein and Nudel control the assembly of the lamin matrix and demonstrate that the lamin matrix also controls the resulting microtubule structures in a feedback loop. Dynein and Nudel are apparent new members of the spindle matrix. The field is now poised to examine how known interactors of Dynein and Nudel, such as dynactin and LIS1, controls the feedback loop of spindle matrix assembly.

\section{References}

1 Scholey, JM, Rogers GC, Sharp DJ. Mitosis, microtubules, and the matrix. J Cell Biol 2001; 154:261-266.

2 Johansen KM, Johansen J. Cell and Molecular Biology of the Spindle Matrix. Int Rev Cytol 2007; 263:155-200.

3 Leslie RJ, Hird RB, Wilson L, McIntosh JR, Scholey JM. Kinesin is associated with a nonmicrotubule component of sea urchin mitotic spindles. Proc Natl Acad Sci USA 1987; 84:2771-2775.

4 Hepler PK, Wolniak SM. Membranes in the mitotic apparatus: their structure and function. Int Rev Cytol 1984; 90:169238.

5 Lince-Faria M, Maffini S, Orr B, et al.

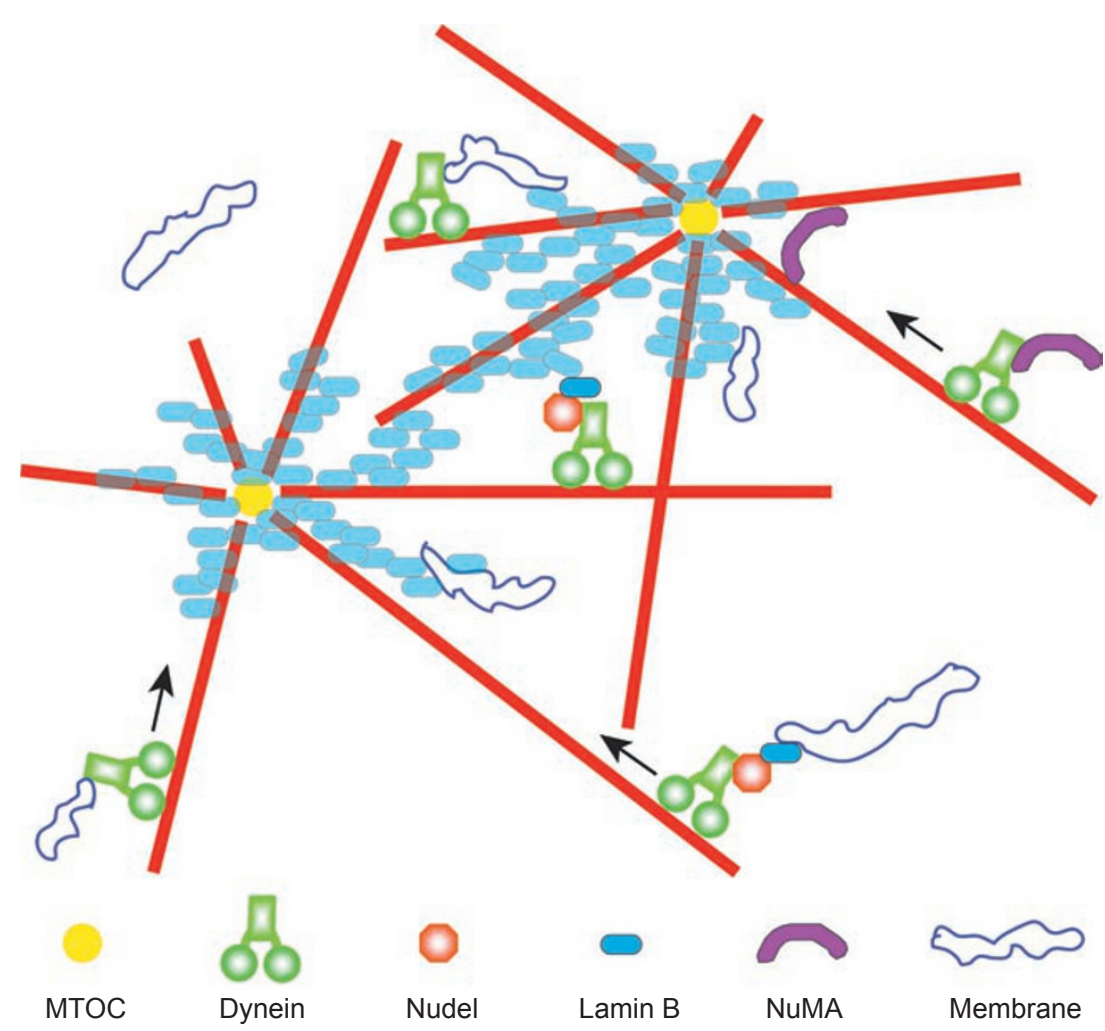

Figure $1 \mathrm{~A}$ model for Dynein and Nudel in the promoting assembly of lamin B matrix during mitosis. 
Spatiotemporal control of mitosis by the conserved spindle matrix protein Megator. J Cell Biol 2009; 184:647-657.

6 Tsai MY, Wang S, Heidinger JM, et al. A mitotic lamin B matrix induced by RanGTP required for spindle assembly. Science 2006; 311:1887-1893.

7 Ma L, Tsai MY, Wang S, et al. Requirement for Nudel and dynein for assembly of the lamin B spindle matrix. Nat Cell Biol 2009; 11:247-256.

8 Efimov VP, Morris NR. The LIS1related NUDF protein of Aspergillus nidulans interacts with the coiled-coil domain of the NUDE/RO11 protein. $J$
Cell Biol 2000; 150:681-688.

9 Li J, Lee WL, Cooper JA. NudEL targets dynein to microtubule ends through LIS1. Nat Cell Biol 2005; 7:686-690.

10 Feng Y, Olson EC, Stukenberg PT, Flanagan LA, Kirschner MW, Walsh CA. LIS1 regulates CNS lamination by interacting with mNudE, a central component of the centrosome. Neuron 2000; 28:665-679.

11 Sweeney KJ, Prokscha A, Eichele G. NudE-L, a novel Lis1-interacting protein, belongs to a family of vertebrate coiled-coil proteins. Mech Dev 2001; 101:21-33.
12 Sasaki S, Shionoya A, Ishida M, et al. A LIS1/NUDEL/cytoplasmic dynein heavy chain complex in the developing and adult nervous system. Neuron 2000; 28:681-696.

13 Niethammer M, Smith DS, Ayala R, et al. NUDEL is a novel Cdk5 substrate that associates with LIS1 and cytoplasmic dynein. Neuron 2000; 28:697-711.

14 Gaetz J, Kapoor TM. Dynein/dynactin regulate metaphase spindle length by targeting depolymerizing activities to spindle poles. J Cell Biol 2004; 166:465471. 